\title{
REALIZATION OF AN X-BAND RF SYSTEM FOR LCLS*
}

\author{
P. McIntosh, R. Akre, W. Brooks, P. Emma and C. Rago, SLAC, 2575 Sand Hill Road, Menlo Park, \\ CA 94025, USA
}

\begin{abstract}
A single X-band (11.424 GHz) accelerating structure is to be incorporated in the LINAC Coherent Light Source (LCLS) design to linearize the energy-time correlation (or gradient) across each bunch; features which originate in the preceding accelerating structures (L0 and L1) [1]. This harmonic RF system will operate near the negative RF crest to decelerate the beam, reducing these non-linear components of the correlation, providing a more efficient compression in the downstream bunch compressor chicanes (BC1 and BC2). These non-linear correlation components, if allowed to grow, would lead to Coherent Synchrotron Radiation (CSR) instabilities in the chicanes, effectively destroying the coherence of the photon radiation in the main LCLS undulator. The many years devoted at SLAC in the development of X-band RF components for the NLC/JLC linear collider project [2], has enabled the technical and financial realization of such an RF system for LCLS. This paper details the requirements for the $\mathrm{X}$-band system and the proposed scheme planned for achieving those requirements.
\end{abstract}

\section{INTRODUCTION}

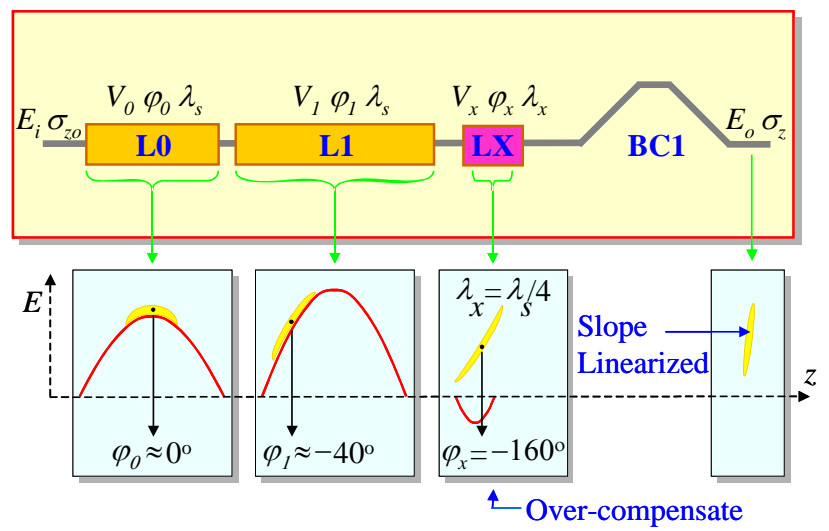

Figure 1: Schematic of two linac segments followed by harmonic RF and compressor chicane. The bunch is compressed from an initial rms length $\sigma_{z 0}$ to a final length $\sigma_{z}$ with initial energy $E_{i}$ and final energy $E_{0}$ (bunch head at left: $z<0$ ).

An X-band $4^{\text {th }}$ harmonic RF section (see Figure 1 ) is to be used to linearize the bunch compression process in the LCLS. The optimum voltage is calculated to compensate both for the second-order RF time-curvature, and for the second-order momentum compaction term of a magnetic bunch compressor chicane. This compensated compression retains, to a much higher degree, the initial temporal distribution of the bunch, reducing the effects of
CSR, and also reducing the sensitivity of the final compression to bunch arrival time variations.

The process of electron bunch compression is usually described as a linear transformation of the longitudinal phase space, which reduces bunch length while increasing energy spread. For future FEL's such as the LCLS, the final compression is so extreme as to be easily dominated by non-linear effects such as sinusoidal RF time-curvature and the compressor's second-order path-length dependence on particle energy. For simple magnetic chicanes and the like, which are ubiquitous in future FEL's, the RF and path-length effects always conspire with the same signed second-order terms to make the problem worse. With increased compression, very sharp temporal spikes can be generated which may drive unwanted collective effects such as CSR and longitudinal wakefields in the main undulator.

The application of a short section of RF accelerating structure at a higher harmonic, with respect to the main accelerating linac RF, can be used to compensate the compression transformation up to second order, thereby maintaining the initial temporal bunch profile and avoiding unnecessary amplification of these undesired collective effects. By linearizing the net transformation, the level of final compression is also made much less sensitive to bunch arrival time errors (or timing jitter).

\section{X-BAND SYSTEM RF REQUIREMENTS}

A decelerating field in the X-band structure will be achieved by operating at $-160^{\circ}$ off the RF crest. To provide this decelerating field, the structure needs to operate at a gradient of $32 \mathrm{MV} / \mathrm{m}$, which equates to a decelerating voltage of $19 \mathrm{MV}$. The beam will then be decelerated from $269 \mathrm{MeV}$ down to $250 \mathrm{MeV}$ before entering into the first bunch compressor chicane BC1. Table 1 shows the X-band RF system requirements for achieving the required deceleration for LCLS.

Table 1: LCLS X-Band RF Requirements.

\begin{tabular}{|l|c|c|}
\hline Parameter & Value & Units \\
\hline Structure Length & 0.6 & $\mathrm{~m}$ \\
\hline Structure Voltage & 19 & $\mathrm{MV}$ \\
\hline Structure Gradient & 32 & $\mathrm{MV} / \mathrm{m}$ \\
\hline RF Phase & -160 & Degrees \\
\hline RF Power at the Structure & 21 & $\mathrm{MW}_{\mathrm{pk}}$ \\
\hline Klystron Output Power & 28 & $\mathrm{MW}_{\mathrm{pk}}$ \\
\hline Repetition Rate & 120 & $\mathrm{~Hz}$ \\
\hline Pulse length & 0.1 & $\mu \mathrm{s}$ \\
\hline
\end{tabular}

*Work supported by DoE contract DE-AC02-76SF00515. 


\section{X-BAND SYSTEM CONFIGURATION}

A single XL-4 klystron [3] will be powered by a modified S-band pulse modulator system, generating up to $410 \mathrm{kV}$ and $350 \mathrm{~A}$ DC. Capable of delivering up to 50 $\mathrm{MW}_{\mathrm{pk}} \mathrm{RF}$ power, the XL-4 klystron will feed in UHV compliant WR90 rectangular waveguide to a circular vacuum system window via a pair of back-to-back mode converter assemblies [4]. These convert from mode $\mathrm{TE}_{10}$ to $\mathrm{TE}_{01}$, through the window and then back to mode $\mathrm{TE}_{10}$ in WR90 rectangular waveguide. The vacuum system window is placed as close as possible to the klystron to avoid unnecessary venting of additional waveguide components whenever the klystron is to be replaced. The RF is again mode converted, this time into WC293 circular waveguide, and transmitted into the beam tunnel. The circular guide provides much lower attenuation compared to WR90 $(0.0035 \mathrm{~dB} / \mathrm{ft}$ compared to $0.03 \mathrm{~dB} / \mathrm{ft})$. Once in the beam tunnel a final conversion is made back to WR90 and the power is then split and supplies the dual $\mathrm{RF}$ feeds of a single $\mathrm{X}$-band accelerating structure mounted on the main LINAC beam axis.

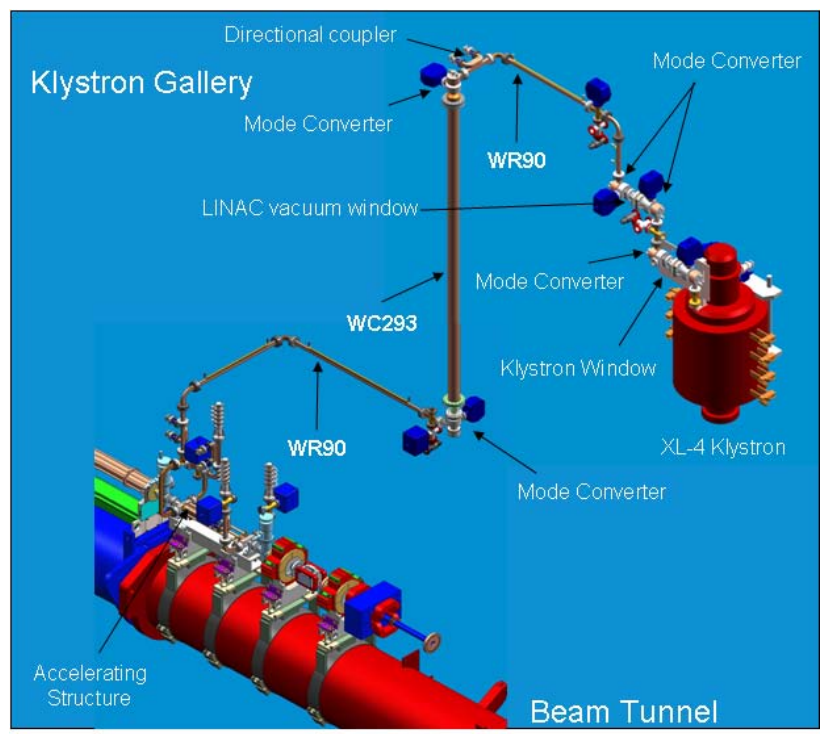

Figure 2: LCLS X-Band RF System Layout.

The total attenuation of the waveguide feed system has been estimated at up to $1.3 \mathrm{~dB}$. With $21 \mathrm{MW}$ needed at the accelerating structure to generate the required accelerating gradient, this will then necessitate a klystron output power of up to $28 \mathrm{MW}$, which is well within the $50 \mathrm{MW}$ capability of this klystron. The round trip group delay of the system has also been estimated as $~ 140$ ns, which for a pulse length operation of 100 ns would mean: if a breakdown occurs in the accelerating structure, the RF power reflected back to the klystron during that same beam pulse, would have extinguished before reaching the klystron output window. This essentially provides isolation for the klystron in the event of a breakdown in the accelerating structure. Successive beam pulses having been inhibited until the breakdown has been overcome.

\section{HARDWARE REALIZATION}

\section{LCLS X-Band Klystron Modulator}

The standard SLAC S-band klystron runs at $350 \mathrm{kV}$, $400 \mathrm{~A}$, and $3.5 \mu$ s flat top. It uses a 15:1 turn ratio pulse transformer which requires the modulator input to be run up to $23 \mathrm{kV}$ at $6 \mathrm{kA}$. Assuming a $2 \mu \mathrm{s}$ rise/fall time, the average power of the modulator is $92 \mathrm{~kW}$. An X-band XL-4 klystron however produces $50 \mathrm{MW}$ running at 410 $\mathrm{kV}$ and $350 \mathrm{~A}$ and by using a pulse transformer with a 17:1 turn ratio in place of the 15:1 unit, the beam voltage can reach up to $400 \mathrm{kV}$ with a $23.5 \mathrm{kV}$ and $6.7 \mathrm{kA}$ input. Again assuming a $2 \mu \mathrm{S}$ rise/fall times (with a $0.15 \mu$ s flat top) the average power will be $40 \mathrm{~kW}$. The higher turn ratio pulse transformer will increase the rise/fall times even further, but it is anticipated that by tuning the pulse forming network of the modulator these increases can be minimized.

\section{LCLS X-Band Klystron}

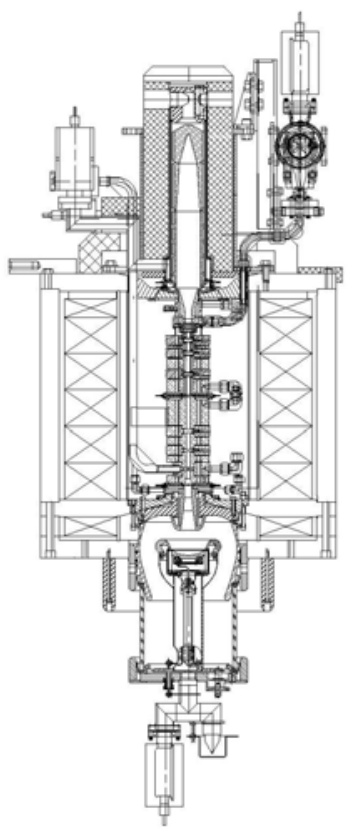

Figure 3: SLAC XL-4 50MW Klystron.

To date, 13 XL-4 X-band klystrons (see Figure 3) have been manufactured at SLAC as part of the NLC/JLC collaboration. Their successful operation up to $50 \mathrm{MW}_{\mathrm{pk}}$ RF power, at repetition rates up to $60 \mathrm{~Hz}$ and pulse lengths out to $1.6 \mu \mathrm{s}$ has made it the obvious choice for the LCLS X-band klystron. At the reduced pulse length, increased repetition frequency, and lower output power for LCLS, compared to typical operating characteristics for an XL-4 at NLCTA where thousands of hours have been put on these tubes (see Table 2), it is not anticipated that the klystron will be performance limited. Operational experience however with the XL-4 klystron at $120 \mathrm{~Hz}$ is minimal, and so plans are underway to high power test an XL-4 at the designated operating parameters for LCLS to verify that there are no operational problems. 
Table 2: Typical XL-4 Operating Parameters in NLCTA.

\begin{tabular}{|l|c|c|}
\hline Parameter & Value & Units \\
\hline Operating Frequency & 11.424 & $\mathrm{GHz}$ \\
\hline RF Pulse Length & 1.5 & $\mu \mathrm{s}$ \\
\hline Peak Output Power & $>50$ & $\mathrm{MW}$ \\
\hline Repetition rate & 60 & $\mathrm{~Hz}$ \\
\hline RF Efficiency & 40 & $\%$ \\
\hline Bandwidth & 120 & $\mathrm{MHz}$ \\
\hline Beam Voltage & 410 & $\mathrm{kV}$ \\
\hline Perveance $\left(10^{-6}\right)$ & 1.2 & \\
\hline Maximum Focusing Field & 4.7 & $\mathrm{kG}$ \\
\hline Gain & 50 & $\mathrm{~dB}$ \\
\hline
\end{tabular}

\section{LCLS X-Band Accelerating Structure}

The $60 \mathrm{~cm}$ long accelerating section (see Figure 4 ) to be used for LCLS is a $150^{\circ}$ phase advance traveling wave structure, developed for the NLCTA accelerator program [5]. The $120 \mathrm{~Hz}$ mode of operation on LCLS does not require the HOM damping features for this structure as the wakefields have sufficient time to naturally decay before the next bunch is decelerated.

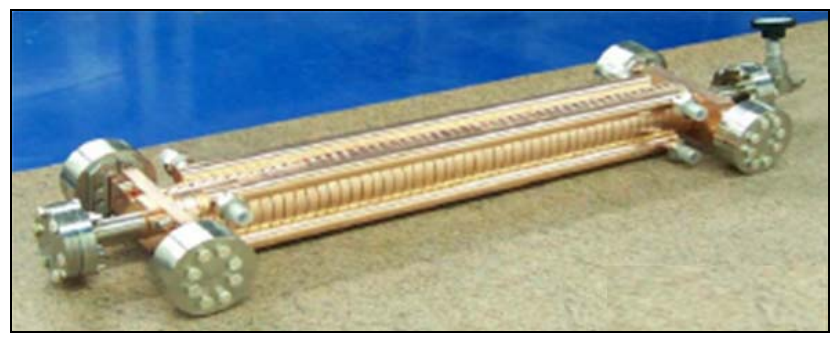

Figure 4: A 60cm NLCTA Accelerating Structure.

This type of accelerating structure has been successfully operated on NLCTA and achieved an accelerating gradient of $65 \mathrm{MV} / \mathrm{m}$ for an RF input of 63 MW. This is then three times that required for LCLS and so it is hoped this will benefit the structures' insensitivity to breakdown events and therefore high reliability can be maintained.

\section{LCLS X-Band Waveguide Components}

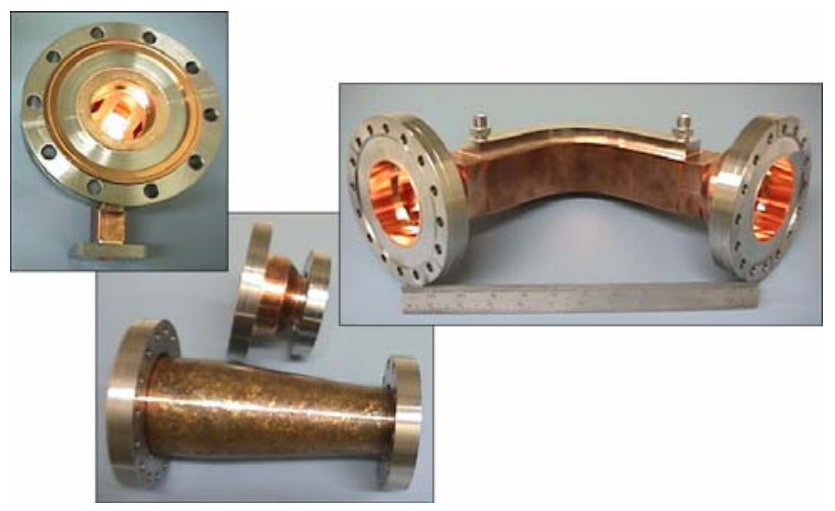

The development of novel waveguide distribution systems (see Figure 5) at X-band for the NLC/JLC collaboration has enabled their direct application for LCLS. E and H-plane bends, mode converters, directional couplers, vacuum pump out sections and various tapers, in particular have all been extensively developed and high power tested on NLCTA at SLAC. LCLS will benefit greatly from this development, as many of these waveguide feeder system components are directly applicable. Reliable operation of the components at $>100$ MW on NLCTA assures system reliability when employed on LCLS.

\section{X-BAND LLRF AND CONTROLS}

Design of the LLRF RF system for the LCLS X-Band station is presently only in the conceptual phase. The idea is to multiply up to X-band from the new low noise SBand LCLS distribution system at the klystron [6]. The 11.424 GHz would then pass through an IQ Modulator for phase and amplitude control. A TWT amplifier would then amplify from the $\mathrm{mW}$ to $\mathrm{kW}$ level to drive the XL-4 klystron. A local phase and amplitude detector will be used to tie into the existing Modulator Klystron Support Unit (MKSU). This local monitor will be used for interlocking of the klystron and supply the main SLAC Control Program (SCP) with phase and amplitude information. Couplers on the input and output of the accelerating structure will be used to supply the LCLS control system with phase and amplitude measurements. These measurements will be used, along with temperature measurements, to hold the accelerator phase and amplitude close to LCLS requirements. Beam-based measurements will then provide adjustments to the L1 SBand accelerators to compensate for non-detectable drifts in the X-Band system.

\section{REFERENCES}

[1] P. Emma, "X-Band RF Harmonic Compensation for Linear Bunch Compression in the LCLS”, LCLS-TN01-1, Nov 2001.

[2] NLC/JLC website at SLAC: http://wwwproject.slac.stanford.edu/lc/local/AccelPhysics/Accel Physics_index.htm

[3] D. Sprehn, et al, "X-Band Klystron Development at the Stanford Linear Accelerator Center”, SPIE 2000 AeroSencse Symposium, Orlando, Florida, April 2000.

[4] S. Tantawi et al, "The Next Linear Collider Test Accelerator's RF Pulse Compression and Transmission Systems”, PAC97, Vancouver, May 1997, pp 3192-3194.

[5] NLCTA website at SLAC: http://wwwproject.slac.stanford.edu/lc/local/Projects/NLCTA/nlc ta.htm

[6] P. McIntosh et al, "Overview of the RF Systems for LCLS”, these proceedings.

Figure 5: SLAC X-Band Waveguide Components. 